\title{
Artrópodes fitófagos e predadores associados em 20 acessos Luffa sp. em sistema orgânico ${ }^{1}$
}

\author{
Phytophagous and predators arthropods associated in 20 accesses of Luffa sp in \\ organic system
}

\author{
Germano Leão Demolin Leite ${ }^{2 *}$, Elwira Daphinn Silva Moreira ${ }^{2}$, Verônica Alves Mota $^{2}$, Izabel Cristina Pereira \\ Vaz Ferreira ${ }^{2}$ e Cândido Alves da Costa ${ }^{2}$
}

\begin{abstract}
Resumo - Os objetivos desta pesquisa foram verificar os artrópodes fitófagos e seus predadores em 20 acessos de bucha vegetal Luffa sp. (Cucurbitaceae), a distribuição destes no dossel das plantas em cultivo orgânico e a produção de frutos dos acessos. O experimento foi em blocos casualizados com 20 tratamentos e quatro repetições. Os tratamentos foram os acessos P159, P304, P466, P03, P570, P569, P467, P30, P446, P495, P125, P263, P644 e P32, provenientes da Embrapa (CENARGEN, DF), e P01, P02, P04, P05, P06 e P07, obtidos em Porteirinha-MG. As parcelas foram compostas por quatro plantas de Luffa sp. A maior abundância de Diabrotica speciosa (Germar) (Coleoptera: Chrysomelidae) foi observada nas folhas do acesso P467 em referência aos demais acessos de Luffa sp. Trigona spinipes (Fabricius) (Hymenoptera: Apidae) foi mais observada nas flores dos acessos P01, P03, P30, P32, P263 e P569 e menos abundantes nos acessos P05, P125, P159, P304, P446 e P570. Os acessos de Luffa sp. com maior número de frutos/planta foram P01, P03, P05, P06, P07, P30, P32, P125, P263, P446 e P570. Os predadores aranhas e Coccinellidae (Coleoptera) bem como o fitófago Cicadellidae (Hemiptera) preferiram atacar a face adaxial, sendo que não se detectou diferença estatística entre as faces para os demais artrópodes. T. spinipes esteve mais presente na parte apical do dossel das plantas. Com esses resultados, o acesso P06 é o mais indicado para o cultivo, pois foi um dos que apresentou maiores produtividades e menores taxas de ataque de insetos.
\end{abstract}

Palavras-chave - Bucha vegetal. Inseto. Inimigos naturais.

\begin{abstract}
The objectives of this research were to verify the phytophagous arthropods and their predators in 20 accesses of vegetable loofah Luffa sp. (Cucurbitaceae), the distribution of the canopy of the plants in organic system and the production of fruits of the accesses. The experiment was in randomized blocks designed with 20 treatments and four replicates. The treatments were the accesses P159, P304, P466, P03, P570, P569, P467, P30, P446, P495, P125, P263, P644 and P32, from Embrapa (CENARGEN, DF, Brazil) and P01, P02, P04, P05, P06 and P07, obtained in Porteirinha, MG, Brazil. Each plot consisted of four plants Luffa sp. The greatest abundance of Diabrotica speciosa (Germar) (Coleoptera: Chrysomelidae) was observed in leaves of the access P467 in reference to the others accessions of Luffa sp.. Trigona spinipes (Fabricius) (Hymenoptera: Apidae) was observed in most flowers of accesses P01, P03, P30, P32, P263 and P569 and less abundant in accesses P05, P125, P159, P304, P446 and P570. The accesses of Luffa sp. highest number of fruits per plant were P01, P03, P05, P06, P07, P30, P32, P125, P263, P446 and P570. The spiders and predatory Coccinellidae (Coleoptera) and the phytophagous Cicadellidae (Hemiptera) preferred to attack the adaxial side, but no statistical difference was detected between the faces to the other arthropods. T. spinipes was present in the apical part of the plant canopy. With these results, access P06 is the most suitable for cultivation, because it was one of those with higher yields and lower rates of insect attack.
\end{abstract}

Key words - Loofah sponge. Insect. Natural enemies.

\footnotetext{
*Autor para correspondência

${ }^{1}$ Recebido para publicação em 11/08/2010; aprovado em 26/05/2011

Pesquisa financiada pelo ICA/UFMG

${ }^{2}$ Insetário George Washington Gomes de Moraes, Universidade Federal de Minas Gerais/Instituto de Ciências Agrárias, Caixa Postal 135, 39.404-006, Montes Claros-MG, Brasil, gldleite@ig.com.br, daphinn@yahoo.com.br, veronicamotabr2000@yahoo.com.br, izabel.vaz@ ig.com.br, candido-costa@ufmg.br
} 


\section{Introdução}

A bucha vegetal (Luffa sp.) (Cucurbitaceae) é proveniente do continente asiático, onde é conhecida e cultivada há séculos. Entre as espécies cultivadas desta família está melancia (Citrullus lanatus Thunb.), abóbora (Cucurbita moschata Duschene), pepino (Cucumis sativus L.), dentre outras que representam uma parcela significativa do agronegócio brasileiro, estimado em R\$ 300 milhões anuais. A Luffa sp. ainda que apresente menor expressão econômica, é a mais cultivada em áreas de agricultura familiar (FERREIRA; DINIZ, 2007).

A agricultura familiar cultiva inúmeros acessos locais de espécies de bucha vegetal, sendo importantes para os pequenos produtores, pois esses utilizam a bucha na confecção de artesanato para comércio em feiras como complementação na renda familiar (FERREIRA; DINIZ, 2007). Os produtores também beneficiam a bucha, de modo que, esta é repassada para o mercado, limpa e embalada.

A cultura da bucha vegetal pode ser danificada por pragas da família das cucurbitáceas, tais como as brocas dos frutos (Diaphania spp.) (Lepidoptera: Crambidae), Dacus cucurbitae Coquillett. (Diptera: Tephritidae) e por ácaros (KOUL; BHAGAT, 1994; VASCONCELOS et al., 2005). Entretanto, a entomofauna associada à bucha vegetal é ainda pouco estudada. As pesquisas sobre a cultura são relacionadas à parte de produção de mudas (MOREIRA et al., 2007), nutrição (SIQUEIRA, 2007) e utilização da bucha vegetal para produção de xaxins (VICHIATO et al., 2008).

Os objetivos desta pesquisa foram verificar os artrópodes fitófagos e seus predadores em 20 acessos de bucha vegetal Luffa sp. (Cucurbitacea), a distribuição destes no dossel das plantas em cultivo orgânico e a produção de frutos destes acessos.

\section{Material e métodos}

O experimento foi conduzido no Instituto de Ciências Agrárias da Universidade Federal de Minas Gerais (ICA/UFMG) em Montes Claros, MG, no período de julho a novembro de 2007. O trabalho foi conduzido numa área experimental de 0,7 ha. $\mathrm{O}$ experimento foi realizado em blocos casualizados com 20 tratamentos e quatro repetições. Os tratamentos foram os acessos: P03, P30, P32, P125, P159, P263, P304, P446, P466, P467, P495, P569, P570 e P644, provenientes da Embrapa (CENARGEN, DF), e P01, P02, P04, P05, P06 e P07, obtidos em PorteirinhaMG. As parcelas eram compostas por quatro plantas de Luffa sp. O sistema de produção adotado foi o orgânico.

As mudas de bucha vegetal foram feitas em copos plásticos $(100 \mathrm{~mL})$ com substrato contendo 3:1 de terra e esterco curtido e acondicionados em casa de vegetação. Após 15 dias, quando a muda atingiu quatro folhas definitivas, foram transplantadas para o campo em covas com dimensões de $20 \times 20 \times 20 \mathrm{~cm}$, preparadas pelo menos 15 dias antes com adubo (20 litros/cova de esterco bovino).

O espaçamento foi de $3 \times 3 \mathrm{~m}$ entre as plantas. As plantas foram conduzidas em haste única, até que atingissem a parte superior da estrutura de arame. $\mathrm{O}$ sistema de irrigação utilizado foi por microaspersão, com turno de rega de dois dias, durante 4 horas.

O levantamento foi realizado em folhas, flores e frutos. No caso das folhas, os artrópodes (fitófagos e predadores) foram contados diretamente (olho nu) semanalmente, avaliando a face adaxial e abaxial de uma folha expandida, escolhida aleatoriamente, em cada parte do dossel (apical, médio e basal) das quatro plantas por parcela. Os artrópodes também foram contabilizados semanalmente, em todas as flores e frutos no dossel dos diferentes acessos de bucha vegetal nas quatro plantas/parcela. Os artrópodes foram coletados e armazenados em recipientes com álcool $70 \%$ e etiquetados contendo dados da coleta (local e data) para futura identificação na coleção de referência do Insetário G.W.G. de Moraes (ICA/UFMG).

Os dados foram submetidos à análise de variância e as médias comparadas pelo teste de médias Scott-Knott, e o coeficiente de correlação de Spearman foi aplicado entre os insetos fitófagos e predadores, ao nível de 5\% de significância.

\section{Resultados e discussão}

Os herbívoros observados nas folhas de bucha vegetal nas seguintes Ordens foram: Coleoptera, Diabrotica speciosa Germar (Chrysomelidae) e Lagria villosa Fabricius (Lagriidae); Hemiptera, Aphis gossypii Glover, Aphis sp. (Aphididae), Bemisia tabaci Genn. (Aleyrodidae), Empoasca sp. (Cicadellidae), Cicadellidae, Pentatomidae, Leptoglossus sp. (Coreidae), Dysdercus spp. (Pyrrhocoridae), Pseudococcus sp. (Pseudococcidae) e Psyllidae; Hymenoptera Trigona spinipes (Fabricius) (Apidae) e Orthoptera Acrididae (TAB. 1). Notaram-se nas flores de bucha vegetal $T$. spinipes e D. speciosa, ambos danificando as flores (TAB. 1). Quanto aos frutos danificados (agente causal desconhecido) os valores não foram expressivos em relação ao ataque das folhas e flores (TAB. 1).

Os insetos fitófagos encontrados sobre Luffa sp. são pragas de outras culturas (GALLO et al., 2002). Contudo, nesse trabalho, se destacaram pelas suas maiores densidades populacionais as cigarrinhas da 
Tabela 1 - Número médio de insetos fitófagos por face foliar, flor ou dano em fruto em variedades de Luffa sp. (Cucurbitaceae) em cultivo orgânico. ICA/UFMG, Montes Claros, MG, 2007

\begin{tabular}{|c|c|c|c|}
\hline Ordem & Família & Espécie & Abundancia $\pm \mathrm{EP}$ \\
\hline \multicolumn{4}{|c|}{ Face foliar - Fitófagos } \\
\hline Coleoptera & Chrysomelidae & Diabrotica speciosa & $0,0188 \pm 0,0019$ \\
\hline Coleoptera & Lagriidae & Lagria villosa & $0,0109 \pm 0,0015$ \\
\hline Hemiptera & Aphididae & Aphis gossypii & $0,0109 \pm 0,0025$ \\
\hline Hemiptera & Aphididae & Aphis sp. & $0,0176 \pm 0,0034$ \\
\hline Hemiptera & Aleyrodidae & Bemisia tabaci & $0,0006 \pm 0,0003$ \\
\hline Hemiptera & Cicadellidae & Empoasca sp. & $0,0012 \pm 0,0004$ \\
\hline Hemiptera & Cicadellidae & Morfoespécie 1 & $0,0571 \pm 0,0110$ \\
\hline Hemiptera & Pentatomidae & Morfoespécie 1 & $0,0014 \pm 0,0006$ \\
\hline Hemiptera & Coreidae & Leptoglossus sp. & $0,0093 \pm 0,0019$ \\
\hline Hemiptera & Pyrrhocoridae & Dysdercus spp. & $0,0093 \pm 0,0018$ \\
\hline Hemiptera & Pseudococcidae & Pseudococcus sp. & $0,0111 \pm 0,0026$ \\
\hline Hemiptera & Psyllidae & Morfoespécie 1 & $0,0058 \pm 0,0010$ \\
\hline Hymenoptera & Apidae & Trigona spinipes & $0,0083 \pm 0,0013$ \\
\hline Orthoptera & Acrididae & Morfoespécie 1 & $0,0188 \pm 0,0043$ \\
\hline \multicolumn{4}{|c|}{ Flor - Fitófagos } \\
\hline Hymenoptera & Apidae & Trigona spinipes & $0,27 \pm 0,01$ \\
\hline Coleoptera & Chrysomelidae & Diabrotica speciosa & $0,0173 \pm 0,0041$ \\
\hline \multicolumn{4}{|c|}{$\begin{array}{l}\text { Fruto - Fitófagos } \\
\end{array}$} \\
\hline $\mathrm{NI}^{*}$ & NI & NI & $0,0058 \pm 0,0058$ \\
\hline
\end{tabular}

família Cicadellidae, o besouro desfolhador D. speciosa, e os pulgões do gênero Aphis nas folhas e, principalmente, a abelha T. spinipes (TAB. 1) que de fato ocasionou danos nas flores na Luffa sp., como observado em outras culturas (GALLO et al., 2002). Entre os Cicadelídeos, a espécie Ricania speculum Walker (Hemiptera), é considerada praga importante nessa cultura nas Filipinas (SOLIS; ESGUERRA, 1982). Isso indica que este grupo de praga deve ser monitorado, pois sugam a seiva das plantas provocando danos diretos e indiretos pela transmissão de toxinas e vírus (GALLO et al., 2002). Crisomelídeos, popularmente conhecidos como vaquinhas, como a Raphidopalpa foveicollis (Fabricius), são consideradas pragas chave desta cultura no oriente (AGARWAL; RASTOGI, 2008), porém esta espécie não foi encontrada no presente trabalho. As brocas das cucurbitáceas, Diaphania hyalinata L. e D. nitidalis Cramer (Lepidoptera: Pyralidae), relatadas como a principal praga desta cultura (SIQUEIRA, 2007) não foram observadas atacando os frutos.

A maioria dos fitófagos observados nas folhas apresentou baixa densidade populacional (TAB. 1). Esse fato talvez se deva à bucha vegetal ser considerada tóxica para alguns animais, como bovinos e eqüinos (SILVA et al., 2006) e sapos (MENON-MIYAKE et al., 2005). Uma das substâncias presentes nas cucurbitáceas é a cucurbitacina. Essa substância é tóxica aos insetos que não coevoluíram com essa família e protege os que coevoluíram contra parasitóides e predadores por armazenarem-na em seus corpos adiposos (METCALF, 1994; TALLAMY et al., 1998).

Dentre os inimigos naturais, foram encontrados nas folhas os predadores joaninhas Cycloneda sanguinea (L.), Eriopsis connexa Germar e Scymnus sp. (Coleoptera: Coccinellidae), Chrysoperla sp. (Neuroptera: Chrysopidae), Zelus sp. (Hemiptera: Reduviidae) e aranhas Miturgidae, Misumenops sp., Oxyopes spp., Theridiidae e Salticidae bem como os protocooperantes Crematogaster sp. e Solenops sp. (Hymenoptera: Formicidae) (TAB. 2). Os inimigos naturais observados neste trabalho são relatados como importantes agentes de controle biológico em diversas culturas (CARVALHO; SOUZA, 2000; GALLO et al., 2002). 
Tabela 2 - Número médio de artrópodes predadores, protocooperantes e polinizadores por face foliar ou flor em variedades de $L u f f a$ sp. (Cucurbitaceae) em cultivo orgânico. ICA/UFMG, Montes Claros, MG, 2007

\begin{tabular}{|c|c|c|c|}
\hline Ordem & Família & Espécie & Abundancia $\pm \mathrm{EP}$ \\
\hline \multicolumn{4}{|c|}{ Face foliar - Predadores } \\
\hline Coleoptera & Coccinellidae & $\begin{array}{c}\text { Cycloneda sanguinea }+ \text { Eriopsis connexa } \\
+ \text { Scymnus } \mathrm{sp} .\end{array}$ & $0,0257 \pm 0,0055$ \\
\hline Neuroptera & Chrysopidae & Chrysoperla sp. & $0,0040 \pm 0,0009$ \\
\hline Hemiptera & Reduviidae & Zelus sp. & $0,0115 \pm 0,0026$ \\
\hline Araneae & $\begin{array}{l}\text { Araneidae, Miturgidae, Thomisidae, } \\
\text { Oxyopidae, theridiidae e Salticidae }\end{array}$ & $\begin{array}{c}\text { Morfoespécies } 1-5,+ \text { Misumenop ssp. }+ \\
\text { Oxyopes spp. }\end{array}$ & $0,0391 \pm 0,0087$ \\
\hline \multicolumn{4}{|c|}{ Face foliar - Protocooperantes } \\
\hline Hymenoptera & Formicidae & Crematogaster sp. + Solenopsis sp. & $0,0328 \pm 0,0054$ \\
\hline \multicolumn{4}{|c|}{ Flor - Polinizadores } \\
\hline Hymenoptera & Apidae & Apis mellifera & $0,0071 \pm 0,0026$ \\
\hline Hymenoptera & Apidae & Tetragonisca angustula & $0,0244 \pm 0,0049$ \\
\hline Hymenoptera & Anthoporidae & Xylocopa sp. & $0,0091 \pm 0,0030$ \\
\hline
\end{tabular}

As correlações entre predadores e insetos fitófagos foram positivas entre as joaninhas e os pulgões $(\mathrm{R}=0,73$; $\mathrm{P}=0,0001) ;$ Chrysoperla $\mathrm{sp}$. com pulgões $(\mathrm{R}=0,71$; $\mathrm{P}=0,0001)$ e com cochonilhas $(\mathrm{R}=0,95 ; \mathrm{P}=0,0001)$ e as aranhas com D. speciosa $(\mathrm{R}=0,26 ; \mathrm{P}=0,0001)$, L. villosa $(\mathrm{R}=0,59 ; \mathrm{P}=0,0001)$ e com cicadelídeos $(\mathrm{R}=0,99$; $\mathrm{P}=0,0001)$. Por outro lado, foi observada protocooperação entre as formigas e os pulgões $(\mathrm{R}=0,76 ; \mathrm{P}=0,0001)$ e as cochonilhas $(\mathrm{R}=0,96 ; \mathrm{P}=0,0001)$. Os pulgões liberam secreções adocicadas, que atraem várias espécies de formigas que dela se alimentam. Muitas espécies de formigas são agressivas, pois atacam outros insetos para defenderem a sua fonte de alimento. Isso contribui para diminuir a infestação destes na planta hospedeira, como observado por Agarwal e Rastogi (2008) em plantações de bucha, onde as formigas controlaram o ataque de $R$. Foveicollis.

Outros himenópteros foram observados nas flores de bucha vegetal além da $T$. spinipes, como os polinizadores Apis mellifera (L.) (Apidae), Tetragonisca angustula Latreille (Apidae) e Xylocopa sp. (Anthophoridae) (TAB. 2). Esses himenópteros podem favorecer a polinização cruzada na bucha vegetal, que constitui numa importante adaptação evolutiva das plantas, aumentando o vigor das espécies, possibilitando novas combinações de fatores hereditários e aumentando a produção de frutos e sementes (COUTO; COUTO, 2002).

Os acessos de Luffa sp. que produziram maior quantidade de frutos foram P01, P03, P05, P06, P07, P30, P32, P125, P263, P446, P496 e P570 comparado com os demais acessos (TAB. 3). Dos seis acessos oriundos da cidade de Porteirinha, norte de Minas Gerais, somente os P02 e P04 não produziram muitos frutos, este fato indica que as demais estão bem adaptadas às condições edafoclimáticas nesta região do estado.

T. spinipes foi mais observada nas flores dos acessos P01, P03, P30, P32, P263 e P569 e menos abundantes nos acessos P05, P125, P159, P304, P446 e P570 (TAB. 3). Provavelmente atributos florais como coloração e o número de flores masculinas (contendo anteras produtoras de pólen) expliquem a atração dessa abelha por determinados acessos de bucha vegetal. Os acessos P03 e P32 apresentam flores com coloração amarela mais intensa que os demais acessos que possuem coloração amarela média (FERREIRA et al., 2008). Já os acessos P569, P30 e P263 apresentaram apenas flores masculinas (FERREIRA et al., 2008). Destes acessos, verificou a maior ocorrência de $T$. spinipes no acesso P263 (TAB. 3). A maior abundância de D. speciosa foi observada nas folhas do acesso P467 (TAB. 3). As causas da maior susceptibilidade da P467 são desconhecidas e serão objetos de estudos futuros.

As abelhas, de forma geral, possuem visão tricromática, enxergando as cores ultravioleta, azul e amarelo, dentro de um espectro de visão que varia de cerca de $336 \mathrm{~nm}$ a $532 \mathrm{~nm}$ (CHITTKA; WALKER, 2006; KEARNS; INOUYE, 1993). Flores de Cosmos sulphureus Cav. Schum. (Asteraceae), de coloração amarelo-alaranjada, são abundantemente visitadas pelas abelhas $A$. mellifera e $T$. spinipes que coletam pólen e néctar (PIRANI; CORTOPASSILAURINO, 1993). No Brasil, Amaral (1970) concluiu que as abelhas Trigona 
Tabela 3 - Número médio de frutos/planta e de adultos de Trigona spinipes/flor e Diabrotica speciosa/face foliar em variedades de Luffa sp. (Cucurbitaceae) em cultivo orgânico. ICA/UFMG, Montes Claros, MG, 2007

\begin{tabular}{crcc}
\hline Variedades & $\mathrm{N}^{\circ}$. de frutos/planta & $\mathrm{N}^{\circ}$. de T. spinipes/flor & $\mathrm{N}^{\circ}$. de D. speciosa/face foliar \\
\hline P01 & $25,50 \mathrm{a}$ & $0,42 \mathrm{a}$ & $0,07 \mathrm{~b}$ \\
P02 & $4,33 \mathrm{~b}$ & $0,22 \mathrm{~b}, 04 \mathrm{~b}$ \\
P03 & $21,09 \mathrm{a}$ & $0,41 \mathrm{a}$ & $0,03 \mathrm{~b}$ \\
P04 & $11,43 \mathrm{~b}$ & $0,24 \mathrm{~b}$ & $0,04 \mathrm{~b}$ \\
P05 & $16,00 \mathrm{a}$ & $0,00 \mathrm{c}$ & $0,00 \mathrm{~b}$ \\
P06 & $23,50 \mathrm{a}$ & $0,26 \mathrm{~b}$ & $0,00 \mathrm{~b}$ \\
P07 & $14,64 \mathrm{a}$ & $0,29 \mathrm{~b}$ & $0,00 \mathrm{~b}$ \\
P30 & $19,00 \mathrm{a}$ & $0,42 \mathrm{a}$ & $0,02 \mathrm{~b}$ \\
P32 & $14,71 \mathrm{a}$ & $0,35 \mathrm{a}$ & $0,00 \mathrm{~b}$ \\
P125 & $14,50 \mathrm{a}$ & $0,06 \mathrm{c}$ & $0,08 \mathrm{~b}$ \\
P159 & $8,70 \mathrm{~b}$ & $0,17 \mathrm{c}$ & $0,02 \mathrm{~b}$ \\
P263 & $22,45 \mathrm{a}$ & $0,45 \mathrm{a}$ & $0,02 \mathrm{~b}$ \\
P304 & $7,33 \mathrm{~b}$ & $0,05 \mathrm{c}$ & $0,00 \mathrm{~b}$ \\
P446 & $15,20 \mathrm{a}$ & $0,16 \mathrm{c}$ & $0,00 \mathrm{~b}$ \\
P466 & $9,27 \mathrm{~b}$ & $0,25 \mathrm{~b}$ & $0,03 \mathrm{~b}$ \\
P467 & $11,09 \mathrm{~b}$ & $0,23 \mathrm{~b}$ & $0,21 \mathrm{a}$ \\
P496 & $15,00 \mathrm{a}$ & $0,29 \mathrm{~b}$ & $0,02 \mathrm{~b}$ \\
P569 & $9,78 \mathrm{~b}$ & $0,42 \mathrm{a}$ & $0,04 \mathrm{~b}$ \\
P570 & $13,00 \mathrm{a}$ & $0,07 \mathrm{c}$ & $0,00 \mathrm{~b}$ \\
P644 & $5,30 \mathrm{~b}$ & $0,30 \mathrm{~b}$ & $0,06 \mathrm{~b}$ \\
\hline
\end{tabular}

As médias seguidas pela mesma letra na coluna não diferem, entre si, pelo teste de média de Scott-Knott ao nível de 1\% de significância

spp. (irapuás) e A. mellifera (africanizadas) foram as mais importantes polinizadoras em cucurbitáceas, tendo encontrado Trigona spp. em maior número na cultura de C. maxima. Convém ressaltar que as flores masculinas predominam nas cucurbitáceas, tendo sido observado maior número de visitas de abelhas também nas flores masculinas (LATTARO; MALERBOSOUZA, 2006), como também relatadas por Ferreira et al. (2008) na bucha vegetal.

Trigona spinipes esteve mais presente na parte apical das plantas (TAB. 4), talvez devido a esta parte do dossel concentrar a maior quantidade de estruturas florais

Tabela 4 - Número médio de Coccinellidae, aranhas e Cicadellidae em função da face foliar e de Trigona spinipes/face foliar em função do dossel de Luffa sp. (Cucurbitaceae) em cultivo orgânico. ICA/UFMG, Montes Claros, MG, 2007

\begin{tabular}{lccc}
\hline & \multicolumn{3}{c}{ Dossel } \\
\cline { 2 - 3 } Trigona spinipes* & Apical & Médio & Basal \\
\cline { 2 - 4 } & $0,014 \mathrm{a}$ & $0,007 \mathrm{~b}$ & $0,004 \mathrm{~b}$ \\
\cline { 2 - 3 } & Face Adaxial & Face abaxial \\
\hline Coccinellidae* & $0,04 \mathrm{a}$ & $0,01 \mathrm{~b}$ \\
Aranhas** & $0,06 \mathrm{a}$ & $0,02 \mathrm{~b}$ \\
Cicadellidae** & $0,08 \mathrm{a}$ & $0,04 \mathrm{~b}$ \\
\hline As médias seguidas pela mesma letra na linha não diferem, entre si, pelo teste de média de Scott-Knott* ao nível de $1 \%$ e ** 5\% de signifiçncia
\end{tabular}


(FERREIRA et al., 2008). Os fitófagos cicadelídeos e os predadores, aranhas e joaninhas, foram encontrados principalmente na face adaxial das folhas da bucha vegetal (TAB. 4), sendo que não se detectou diferença estatística entre as faces para os demais artrópodes. De forma geral, a maior incidência de fitófagos e de seus inimigos naturais ocorreu no início do cultivo da bucha vegetal (TAB. 5). Por outro lado, os insetos observados nas flores de bucha vegetal ocorreram mais em setembro, outubro e novembro, período de maior florescimento (TAB. 5).
Os insetos, com destaque para os sugadores, atacam preferencialmente as folhas apicais, principalmente a face inferior no início do cultivo, por estas serem mais tenras, com menores quantidades de cálcio e de fibras, e de alto valor nutricional com alto teor de nitrogênio (CHAU et al., 2005; LEITE et al., 2002, 2005; SANTOS et al., 2003; SILVA et al., 1998). Em contraste, no presente trabalho foi observado maior ataque de cigarrinhas na face superior, provavelmente devido à maior presença de tricomas na face inferior.

Tabela 5 - Número médio de insetos e de aranhas/face foliar ou em flor Luffa sp. (Cucurbitaceae) em cultivo orgânico em função dos meses. ICA/UFMG, Montes Claros, MG, 2007

\begin{tabular}{|c|c|c|c|c|c|}
\hline \multicolumn{6}{|c|}{ Face foliar } \\
\hline Artrópodes & Julho & Agosto & Setembro & Outubro & Novembro \\
\hline Diabrotica speciosa & $0,04 \mathrm{a}$ & $0,01 \mathrm{~b}$ & $0,03 \mathrm{a}$ & $0,01 \mathrm{~b}$ & $0,01 \mathrm{~b}$ \\
\hline Lagria villosa & $0,02 \mathrm{a}$ & $0,01 \mathrm{~b}$ & $0,03 \mathrm{a}$ & $0,00 \mathrm{~b}$ & $0,00 \mathrm{~b}$ \\
\hline Dysdercus sp. & $0,04 \mathrm{a}$ & $0,00 \mathrm{~b}$ & $0,00 \mathrm{~b}$ & $0,00 \mathrm{~b}$ & $0,01 \mathrm{~b}$ \\
\hline Aphis gossypii & $0,06 \mathrm{a}$ & $0,00 \mathrm{~b}$ & $0,00 \mathrm{~b}$ & $0,00 \mathrm{~b}$ & $0,00 \mathrm{~b}$ \\
\hline Aphis sp. & $0,06 \mathrm{a}$ & $0,00 \mathrm{~b}$ & $0,02 \mathrm{~b}$ & $0,01 \mathrm{~b}$ & $0,00 \mathrm{~b}$ \\
\hline Pseudococcus sp. & $0,06 \mathrm{a}$ & $0,00 \mathrm{~b}$ & $0,00 \mathrm{~b}$ & $0,00 \mathrm{~b}$ & $0,00 \mathrm{~b}$ \\
\hline Leptoglossus sp. & $0,04 \mathrm{a}$ & $0,00 \mathrm{~b}$ & $0,00 \mathrm{~b}$ & $0,01 \mathrm{~b}$ & $0,00 \mathrm{~b}$ \\
\hline Psyllidae & $0,00 \mathrm{~b}$ & $0,00 \mathrm{~b}$ & $0,02 \mathrm{a}$ & $0,00 \mathrm{~b}$ & $0,00 \mathrm{~b}$ \\
\hline Cicadellidae & $0,27 \mathrm{a}$ & $0,00 \mathrm{~b}$ & $0,01 \mathrm{~b}$ & $0,02 \mathrm{~b}$ & $0,02 \mathrm{~b}$ \\
\hline Trigona spinipes & $0,02 \mathrm{a}$ & $0,00 \mathrm{~b}$ & $0,01 \mathrm{~b}$ & $0,01 \mathrm{~b}$ & $0,00 \mathrm{~b}$ \\
\hline Acrididae & $0,11 \mathrm{a}$ & $0,00 \mathrm{~b}$ & $0,00 \mathrm{~b}$ & $0,00 \mathrm{~b}$ & $0,00 \mathrm{~b}$ \\
\hline Tettigonidae & $0,04 \mathrm{a}$ & $0,00 \mathrm{~b}$ & $0,00 \mathrm{~b}$ & $0,00 \mathrm{~b}$ & $0,00 \mathrm{~b}$ \\
\hline Aranhas & $0,21 \mathrm{a}$ & $0,01 \mathrm{~b}$ & $0,00 \mathrm{~b}$ & $0,00 \mathrm{~b}$ & $0,00 \mathrm{~b}$ \\
\hline Coccinellidae & $0,14 \mathrm{a}$ & $0,00 \mathrm{~b}$ & $0,00 \mathrm{~b}$ & $0,00 \mathrm{~b}$ & $0,00 \mathrm{~b}$ \\
\hline Lampyridae & $0,04 \mathrm{a}$ & $0,00 \mathrm{~b}$ & $0,01 \mathrm{~b}$ & $0,00 \mathrm{~b}$ & $0,00 \mathrm{~b}$ \\
\hline Zelus sp. & $0,06 \mathrm{a}$ & $0,00 \mathrm{~b}$ & $0,00 \mathrm{~b}$ & $0,00 \mathrm{~b}$ & $0,00 \mathrm{~b}$ \\
\hline Formicidae & $0,13 \mathrm{a}$ & $0,00 \mathrm{~b}$ & $0,02 \mathrm{~b}$ & $0,01 \mathrm{~b}$ & $0,01 \mathrm{~b}$ \\
\hline Chysoperla sp. & $0,02 \mathrm{a}$ & $0,00 \mathrm{~b}$ & $0,00 \mathrm{~b}$ & $0,00 \mathrm{~b}$ & $0,00 \mathrm{~b}$ \\
\hline Sphecidae & $0,04 \mathrm{a}$ & $0,00 \mathrm{~b}$ & $0,00 \mathrm{~b}$ & $0,00 \mathrm{~b}$ & $0,00 \mathrm{~b}$ \\
\hline Tiphiidae & $0,04 \mathrm{a}$ & $0,00 \mathrm{~b}$ & $0,00 \mathrm{~b}$ & $0,00 \mathrm{~b}$ & $0,00 \mathrm{~b}$ \\
\hline Pompilidae & $0,06 \mathrm{a}$ & $0,00 \mathrm{~b}$ & $0,00 \mathrm{~b}$ & $0,00 \mathrm{~b}$ & $0,00 \mathrm{~b}$ \\
\hline \multicolumn{6}{|c|}{ Flor } \\
\hline Diabrotica speciosa & $0,00 \mathrm{~b}$ & $0,00 \mathrm{~b}$ & $0,06 \mathrm{a}$ & $0,01 \mathrm{~b}$ & $0,01 \mathrm{~b}$ \\
\hline Trigona spinipes & $0,10 \mathrm{c}$ & $0,29 \mathrm{~b}$ & $0,48 \mathrm{a}$ & $0,32 \mathrm{~b}$ & $0,07 \mathrm{c}$ \\
\hline Tetragonisca angustula & $0,00 \mathrm{~b}$ & $0,00 \mathrm{~b}$ & $0,02 \mathrm{~b}$ & $0,04 \mathrm{a}$ & $0,06 \mathrm{a}$ \\
\hline Xylocopa sp. & $0,00 \mathrm{~b}$ & $0,00 \mathrm{~b}$ & $0,01 \mathrm{~b}$ & $0,03 \mathrm{a}$ & $0,02 \mathrm{a}$ \\
\hline Apis mellifera & $0,00 \mathrm{a}$ & $0,00 \mathrm{a}$ & $0,02 \mathrm{a}$ & $0,01 \mathrm{a}$ & $0,01 \mathrm{a}$ \\
\hline
\end{tabular}

As médias seguidas pela mesma letra na linha não diferem, entre si, pelo teste de média de Scott-Knott ao nível de 1\% de significância 


\section{Conclusões}

1.Os principais insetos fitófagos nas folhas foram os cicadelídeos e crisomelídeos e nas flores T. spinipes;

2. Os principais predadores são os coccinelídeos e as aranhas;

3.O acesso mais indicado para cultivo comercial é o P06 devido ao maior número de frutos/planta e menor incidência de insetos fitófagos.

\section{Referências}

AGARWAL, V. M.; RASTOGI, N. Deterrent effect of a guild of extrafloral nectary-visiting ant species on Raphidopalpa foveicollis, a major insect pest of sponge gourd, Luffa cylindrica. Entomologia Experimentalis et Applicata, v. 128, n. 02, p. 303-311, 2008.

AMARAL, G. Estudos de polinização entomófila de plantas de interesse econômico para o Brasil, realizados na Escola Superior de Agricultura "Luiz de Queiroz". In: CONGRESSO BRASILEIRO DE APICULTURA, 1., 1970, Florianópolis. Anais... Florianópolis. 1970. p. 65-68.

CARVAlHO, C. F.; SOUZA, B. Métodos de criação e produção de crisopídeos. In: BUENO, V. H. P. (Ed.). Controle biológico de pragas: produção massal e controle de qualidade. Lavras: UFLA, 2000. p. 91-109.

CHAU, A. et al. Influences of fertilization on Aphis gossypii and insecticide usage. Journal of Applied Entomology, v. 129, n. 02, p. 89-97, 2005.

CHITTKA, L.; WALKER, J. Do bees like Van Gogh's Sunflowers? Optics \& Laser Technology, v. 38, p. 323-328, 2006.

COUTO, R. H. N.; COUTO, L. A. Apicultura: manejo e produtos. 2. ed. Jaboticabal: FUNEP, 2002. 191 p.

FERREIRA, M. A. F.; DINIZ, F. Rede de pesquisa vai incrementar a produção de cucurbitáceas em áreas de agricultura familiar e assentamentos. 2007. Disponível em: <http://www.infobibos.com/ Artigos/2007_3/curcubitaceas/index.htm>.acesso em: 11 jan. 2010.

FERREIRA, I. C. P. V. et al. Caracterização morfológica de acessos de bucha vegetal (Luffa spp.). In: CONGRESSO NACIONAL DE BOTÂNICA, 59., 2008, Natal. Anais... Natal. 2008. 1 CD-ROM.

GALLO, D. et al. Manual de entomologia agrícola. Piracicaba: FEALQ, 2002. $920 \mathrm{p}$.

KEARNS, C. A.; INOUYE, D. W. Techniques for pollination biologists. Niwot: University Press of Colorado, 1993. 583 p.

KOUL, V. K.; BHAGAT, K. C. Effect of host plants on the developmental stages of the fruit fly, Dacus cucurbitae Coquillet. Annals of Plant Protection Sciences, v. 02, n. 02, p. 8-11, 1994.

LATTARO, L. H.; MALERBO-SOUZA, D. T. Polinização entomófila em abóbora caipira, Cucurbita mixta (Curcubitaceae). Acta Scientiarum Agronomy, v. 28, n. 04, p. 563-568, $2006 \mathrm{a}$.
LEITE, G. L. D. et al. Effect of leaf characteristics, natural enemies and climatic conditions on the intensities of Myzus Persicae and Frankliniella schulzei attacks on Lycopersicon esculentum. Arquivos do Instituto Biológico, v. 69, n. 04, p. 71-82, 2002.

LEITE, G. L. D. et al. Bemisia tabaci, Brevicoryne brassicae and Thrips tabaci abundance on Brassica oleracea var. acephala. Pesquisa Agropecuária Brasileira, v. 40, n. 03, p. 197-202, 2005.

MENON-MIYAKE, M. A. et al. Luffa operculata effects on the epithelium of frog palate: histological features. Revista Brasileira de Otorrinolaringologia, v. 71, n. 02, p. 132$138,2005$.

METCALF, R. L. Chemical ecology of Diabroticites. In: JOLIVET, P. H. et al. (Ed). Novel aspects of the biology of Chrysomelidae. The Hague: Kluwer Academic Publishers, 1994. p. 153-169.

MOREIRA, F. J. C. et al. Emergência e crescimento inicial de plântulas de bucha (Luffa cylindrica Roemer). Revista Ciência Agronômica, v. 38, n. 02, p. 169-175, 2007.

PIRANI, J. R.; CORTOPASSI-LAURINO, M. Flores e abelhas em São Paulo. São Paulo: EDUSP/FAPESP, 1993. 192 p.

SANTOS, T. M. et al. Influência de tricomas do algodoeiro sobre os aspectos biológicos e capacidade predatória de Chrysoperla externa (Hagen) alimentada com Aphis gossypii Glover. Bragantia, v. 62, n. 02, p. 243-254, 2003.

SILVA, D. M. et al. Plantas tóxicas para ruminantes e eqüídeos no Seridó Ocidental e Oriental do Rio Grande do Norte. Pesquisa Veterinária Brasileira, v. 26, n. 04, p. 223-236, 2006.

SILVA, C. C. et al. Comparison of leaf chemical composition and attack patterns of Tuta absoluta (Meyrick) (Lepidoptera: Gelechiidae) in Three tomato species. Agronomia Lusitana, v. 46, n. 02/04, p. 61-71, 1998.

SIQUEIRA, R. G. Crescimento e acúmulo de nutrientes em bucha vegetal (Luffa cylindrica). 2007. 48 f. Dissertação (Mestrado em Fitotecnia) - Universidade Federal de Viçosa, Viçosa.

SOLIS, A. D.: ESGUERRA, N. M. Biology of the black leafhopper, Ricania speculum Walker on patola (Luffa cylindrica (L.) Roem). Annals of Tropical Research, v. 04, n. 04, p. 259-267, 1982.

TALLAMY,D. W.etal. Sequestered cucurbitacins and pathogenicity of Metarhizium anisopliae (Moniliales: Moniliaceae) on spotted cucumber beetle eggs and larvae (Coleoptera: Chrysomelidae). Environmental Entomology, v. 27, p. 366-372, 1998.

VASCONCELOS, G. J. N. et al. Ocorrência de Eriophyoidea, Tenuipalidae, Tarsonemidae e Tukerellidae (Acari) em fruteiras no Estado de Pernambuco, Brasil. Caatinga, v. 18, n. 02, p. 98-104, 2005.

VICHIATO, M. R. M. et al. Bucha vegetal e fertilização organomineral no cultivo de Dendrobium nobile Lindl. Revista da FZVA, v. 15, n. 01, p. 34-42, 2008. 